\title{
Routine Versus Selective Use of Chest and Abdominopelvic CT-Scan in Blunt Trauma: A Randomized Controlled Study
}

\author{
Hamidreza Talari, ${ }^{1, *}$ Nushin Moussavi, Abdolhossein Davoodabadi, Sasan Saidfar, and Fatemeh Atoof \\ ${ }^{1}$ Kashan University of Medical Sciences, Kashan, Iran \\ "Corresponding author: Hamidreza Talari. E-mail: talari2008hr@yahoo.com \\ Received 2016 December 21; Accepted 2017 February 08.
}

\begin{abstract}
Background: Whole body CT-scan (WBCT) is increasingly used in the setting of severe blunt trauma due higher sensitivity for detecting occult injuries in comparison to conventional radiography and ultrasound. But the real impact of routine CT-scan on patient outcome is still unclear. Considering the high radiation exposure due to routine CT-scan, this study was conducted to assess the effect of performing routine chest and abdominopelvic CT-scan on patient admission time and complications in blunt trauma.

Methods: One hundred and forty conscious and hemodynamically stable blunt trauma patients were included in this randomized controlled study. For seventy patients, routine chest and abdominopelvic CT-scan was requested in addition to conventional radiography and ultrasound. For the other 70 patients, selective CT-scan was ordered according to the clinical presentation. Patient admission times in the emergency room and surgery ward, complications and surgical procedures were assessed. "Missed injuries" defined as additional findings on CT-scan which were not evident on radiography or ultrasound were assessed.

Results: Admission time in the emergency ward was significantly shorter in the intervention group with $3.7 \pm 0.9$ hours in comparison to $4.9 \pm 0.8$ hours in the control group. Also mean admission time in hospital was only $5.4 \pm 2.4$ in the intervention group in comparison to $7 \pm 3.3$ days in the control group. On abdominopelvic CT-scan, 9 (7.8\%) additional injuries were detected. All these 9 patients had a positive clinical examination. Chest CT-scan in the intervention group led to additional diagnoses in 17 patients (24.28\%) leading to tube thoracostomy insertion in 13 patients (18.57\%).

Conclusions: Performing routine chest and abdominopelvic CT-scan in conscious blunt trauma patients decreases the hospitalization time but has no effect on patient complications and final outcome. Using a selective approach and performing CT-scan only for symptomatic patients, would be an appropriate option to decrease radiation dose and facility overuse. Nevertheless, the concern of missing occasionally a life threatening injury still remains. Each trauma center should make the decision whether to perform CT-scan or not according to all the pros and contras of this imaging modality.
\end{abstract}

This is an abstract presented in the 33rd Iranian congress of radiology (ICR) and the 15th congress of Iranian radiographic science association (IRSA). 